\title{
Social network theory: new insights and issues for behavioral ecologists
}

\author{
Andrew Sih • Sean F. Hanser • Katherine A. McHugh
}

Received: 12 December 2007 /Revised: 5 February 2009 / Accepted: 5 February 2009/Published online: 2 April 2009

(C) The Author(s) 2009. This article is published with open access at Springerlink.com

\begin{abstract}
Until recently, few studies have used social network theory (SNT) and metrics to examine how social network structure (SNS) might influence social behavior and social dynamics in non-human animals. Here, we present an overview of why and how the social network approach might be useful for behavioral ecology. We first note four important aspects of SNS that are commonly observed, but relatively rarely quantified: (1) that within a social group, differences among individuals in their social experiences and connections affect individual and group outcomes; (2) that indirect connections can be important (e.g., partners of your partners matter); (3) that individuals differ in their importance in the social network (some can be considered keystone individuals); and (4) that social network traits often carry over across contexts (e.g., SN position in male-male competition can influence later male mating success). We then discuss how these four points, and the social network approach in general, can yield new
\end{abstract}

Communicated by J. Krause

This contribution is part of the special issue "Social networks: new perspectives" (Guest Editors: J. Krause, D. Lusseau and R. James).

A. $\operatorname{Sih}(\bowtie)$

Department of Environmental Science and Policy,

University of California at Davis,

Davis, CA 95616, USA

e-mail: asih@ucdavis.edu

\section{S. F. Hanser}

Department of Veterinary Medicine: Population Health and Reproduction, University of California at Davis,

Davis, CA 95616, USA

K. A. McHugh

Animal Behavior Graduate Group,

University of California at Davis,

Davis, CA 95616, USA insights and questions for a broad range of issues in behavioral ecology including: mate choice, alternative mating tactics, male-male competition, cooperation, reciprocal altruism, eavesdropping, kin selection, dominance hierarchies, social learning, information flow, social foraging, and cooperative antipredator behavior. Finally, we suggest future directions including: (1) integrating behavioral syndromes and SNT; (2) comparing space use and SNS; (3) adaptive partner choice and SNS; (4) the dynamics and stability (or instability) of social networks, and (5) group selection shaping SNS.

Keywords Social network theory - Social behavior . Mate choice $\cdot$ Cooperation $\cdot$ Dominance hierarchies

\section{Introduction}

Recent years have seen a surge of interest in using network theory to analyze patterns of interactions among "nodes" of various sorts (Newman 2003a; Proulx et al. 2005) including gene or protein networks (Lee et al. 2002; Barabási and Oltvai 2004; Hahn and Kern 2005; Stumpf et al. 2007), neural networks (Loughlin and Sejnowski 2003; Sporns and Kotter 2004; Humphries et al. 2006), networks of interacting individual organisms (Scott 2000; Lusseau and Newman 2004; Lusseau et al. 2006; McCowan et al. 2008), and networks of interacting species (Dunne et al. 2002; Bascompte et al. 2006; Olesen et al. 2006). Social scientists have a long history of examining human interactions using social network theory (SNT; reviewed in Scott 2000). Relatively few studies, however, have used well-established SNT tools to examine social behavior or social dynamics in non-human animals (Wey et al. 2007; Croft et al. 2008). To date, most examples of the use of SNT in non-human 
animals have focused on primates (Flack et al. 2006; Krause et al. 2007; McCowan et al. 2008). However, this special issue of Behavioral Ecology and Sociobiology and the recent symposium at the IEC 2007 conference illustrate the growing recognition of the potential value of using SNT to examine how social network structure (SNS) might influence social dynamics in any organism with social interactions (Krause et al. 2007, 2009; Wey et al. 2007; Croft et al. 2008). The goal of this paper is to help promote the use of SNT in animal behavior by presenting a conceptual overview on how and why social network analyses might be useful in generating new insights for behavioral ecologists. We discuss some key general points about the importance of social structure that can be quantified using social network (SN) metrics and apply these points to mainstream issues in behavioral ecology. We illustrate these points with some recent published work using SN metrics. Finally, we suggest some new issues for behavioral ecologists to study, stimulated by the SNT framework.

\section{General issues about social structure}

The basic premise underlying the value of SNT is that social structure matters, where social structure quantifies the pattern of interactions and relationships among individuals in a social group (Hinde 1976; Whitehead 1997). SN metrics provide a method for quantifying social network structure (SNS). SNS matters for behavioral ecology in that the group's SNS and the individual's social network position can have important effects on social dynamics, individual fitness, and group function and can thus affect the evolution of social behavior.

The fact that SNS matters is not new to animal behaviorists. Accordingly, the issues that we discuss below are not new to animal behavior. Instead, our view is that SNT offers a framework for quantifying aspects of social structure that have long been part of animal behaviorists' intuitive thinking but have often not been quantified in much detail. In particular, we suggest that the following four general aspects of SNS are common and often important: (1) individuals differ in their social experiences; (2) indirect connections matter; (3) individuals differ in their importance in the social network; and (4) social network connections in one context carry over to influence social dynamics in other contexts.

\section{Individuals differ in their social experiences}

Although it is often obvious that individuals differ in their social experiences, much of the theory and empirical traditions in behavioral ecology ignores this simple fact.
For example, simple game models typically assume either that all individuals interact with all others in the population, or if there are two (or more) types, that all individuals experience the average frequency of each type. In simple hawk-dove models, if the population is made up of a mix of $50 \%$ pure hawks and $50 \%$ pure doves, an implicit assumption (e.g., in calculating the fitness of hawks and doves) is that both hawks and doves experience $50 \%$ hawks and $50 \%$ doves. In reality, of course, individuals often interact only with a subset of the overall population, and might thus vary substantially in their social experiences. Some hawks might interact primarily with hawks, while others interact primarily with other doves. Next, we outline some general ways that variation in the actual pattern of social experiences should affect fitness and potentially social evolution.

A fundamental SN metric is the individual's degree $=$ number of social partners. This metric is a major focus of studies of sexual selection; a male's mating degree, his number of mating partners, is mating success. In contrast, although existing theory and intuition suggests that variation in the number of social partners is likely to be important in most social contexts, outside of the male's mating degree, individual variation in degree is usually not discussed. In theory, for example, the number of encounters that a female has with high quality males should affect her choosiness (her tendency to reject low quality males). In Fig. 1, we show the scenario where the average female experiences enough high quality males to be choosy. If all females are average females (if there was no SN structure),

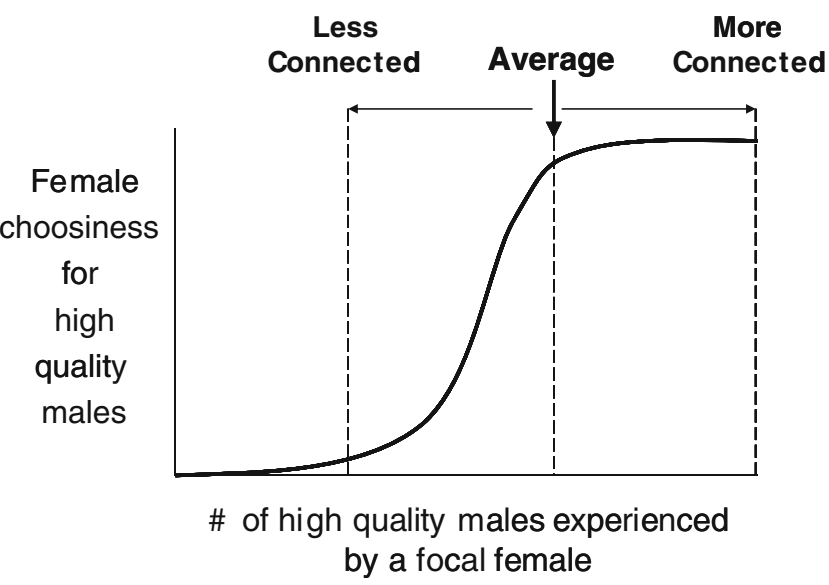

Fig. 1 An example of how social network structure (SNS) can affect adaptive behavior, in this case, female mate choice. If, on average, females experience numerous high quality males, females should be choosy and sexual selection should be strong. However, in real social networks, females typically vary in their encounter frequency with high quality males. Females that encounter few high quality males should not be choosy. Even if, on average, females encounter enough high quality males to be choosy, if the SNS is such that a sizable number of females do not encounter many high quality males, sexual selection can be greatly reduced 
then all females should be choosy and sexual selection should be very strong. If, however, females vary substantially in the number of high quality males encountered, then some, perhaps many, females should not be choosy and sexual selection should be concomitantly weaker. The simple point is that the female-male SNS could have major impacts on mating dynamics and sexual selection. For another example, at both an individual and a group level, the importance of an aggressive dominant individual (or of a cooperator) likely depends on whether the dominant individual (or cooperator) has a large or small degree.

When a population has two or more behavioral types, a key aspect of SNS can be the strategy correlation, the correlation between an individual's behavioral type (BT) or strategy and the frequency with which it interacts with other BTs or strategies. For example, the fitness of hawks and doves is clearly very different if instead of interacting randomly, the group exhibits a positive strategy correlation where doves interact primarily with doves (e.g., if doves successfully avoid hawks) and hawks interact primarily with other hawks. Similarly, for the evolution of cooperation, a key is the frequency with which altruists encounter other altruists. Indeed, it has been noted that what all theories of altruism (kin selection, reciprocal altruism, group selection) have in common is the importance of the covariance between the tendency to be altruistic and the frequency of other altruists encountered (McElreath and Boyd 2007).

\section{Indirect connections matter}

SNS can be particularly interesting when we consider not just direct connections among individuals, but also, indirect connections. The idea is that outcomes can depend on not just who a focal interacts with, but also with whom the focal's partners interact. Going just one step beyond direct partners, relevant, commonly calculated social network metrics include: the focal's reach (the number of friends of friends, the number of individuals connected to a focal via two steps), and the clustering coefficient (the tendency for a focal's friends to also be friends with each other) (Newman 2003a; Croft et al. 2008). These concepts are illustrated in Fig. 2.

A new, indirect $\mathrm{SN}$ metric (introduced here) that should be valuable for many issues in animal behavior is exclusivity = $1 /$ (the degree of a focal's partners). If partner A interacts only with the focal, the focal has an exclusive relationship with A, whereas if $\mathrm{A}$ also interacts with three other individuals, then exclusivity is only $1 / 4$ (see Fig. 2). If the focal individual gets net positive effects from a partner (e.g., predators from prey, or beneficial acts from a cooperator), then lower exclusivity dilutes these positive effects. In sexual selection, the norm is to equate a male's mating degree with his mating success. However, if a male, on average, mates with females

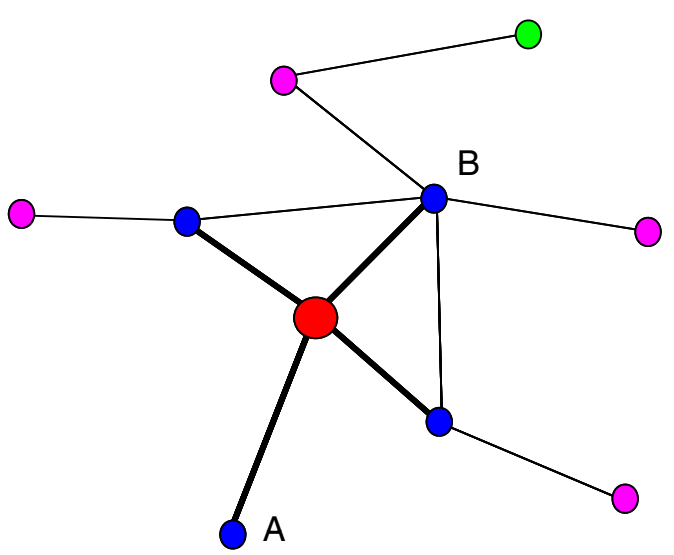

- Connections between the focal individual and direct interactants

- Connections between non-focal individuals

Fig. 2 A simple network illustrating social network metrics. Circles (nodes) are individuals. Lines indicate interactions (referred to as edges). The red node is a focal individual. It has degree $=4$ because it interacts directly with four other individuals, the blue nodes. Individual A has degree $=1$ and $B$ has degree $=5$. The focal has a reach $=4$, because it connects to four individuals, the pink nodes, along pathways of two edges. The green node is outside of the focal's reach. The focal has an exclusive relationship with $\mathrm{A}$ but has an exclusivity value of $1 / 5(0.2)$ for its relationship with $B$, because $B$ interacts with four other individuals besides the focal. The clustering coefficient for the focal is 0.33 because two of the six possible connections among its direct neighbors exist in the network

who mate with many other males, then this low exclusivity can substantially reduce the benefit of his high mating success. If males and females exhibit a positive male-female degree correlation (males that mate with many females tend to mate with females who also mate with numerous other males) then this should substantially reduce overall sexual selection. Conversely, a negative male-female degree correlation (where males with high mating success also enjoy high exclusivity, while numerous males that mate with only one female mate with the same female) should increase sexual selection. Although exclusivity and the male-female degree correlation clearly relate to sperm competition and to extrapair copulations, these "two-step" SN metrics are rarely shown in published papers.

A less familiar SN concept involves an individual's betweenness or centrality (the two metrics are statistically similar) $=$ the proportion of shortest pathways between all individuals in the group that go through a given individual. In intuitive terms, an individual has particularly high centrality if it is the only bridge between two subgroups. For issues regarding flow (e.g., of information or disease), centrality can be useful for identifying critical individuals that control flow or relationships between groups (Granovetter 1973; Gould and Fernandez 1989; Wey et al. 2007).

Some ideas on potentially important indirect connections can be drawn from analogs in community ecology where 
indirect effects have long been thought to be important. For example, trophic cascades occur when carnivores have positive effects on plants via their negative effects on herbivores (Polis and Strong 1996; Yodzis 2000). Similarly in dominance hierarchies, behavioral cascades might involve high-rank individuals having positive effects on low-rank ones by suppressing individuals of intermediate rank. Beyond identifying types of indirect effects per se, by analogy with community ecology, it would be interesting to partition out the relative importance of direct and indirect pathways in governing the overall interaction between pairs of individuals. The overall group's SNS and a focal individual's SN position should have quantifiable effects on the relative importance of these direct and indirect social interactions.

Individuals differ in their importance in the social networkkeystone individuals

Anecdotally, it seems obvious that in any given social group, individuals differ in the magnitude of their effects on other individuals and/or on overall group function (Wey et al. 2007). Few studies, however, have explicitly quantified these differences, their determinants, or their consequences (but see Flack et al. 2005; Flack et al. 2006). Sih and Watters (2005) introduced the notion of a keystone individual drawn by analogy from community ecology's keystone species. Keystone individuals have a disproportionately large effect on the overall group's dynamics or function. An obvious example might be the alpha individual in a group. However, whereas alpha status is usually evaluated by an animal's tendency to win contests, the importance of an alpha individual should depend on its pattern of interaction (direct and indirect) with others in the group. An alpha individual will likely be more important if it has a high degree, high reach, or perhaps high centrality. The same notion - that importance depends on SN traitsshould apply for other potentially important positions - e.g., conflict mediators (Flack et al. 2006) or hyper-aggressive individuals (Sih and Watters 2005).

As noted earlier, another obvious type of keystone individual is one who controls flow through a social group - e.g., flow of information (Gould and Fernandez 1989) or flow of disease (Meyers et al. 2005; Rothenberg and Muth 2007). SNT has been used extensively in the social sciences to analyze how SNS (e.g., the organizational structure of a business, university department, or military hierarchy) influences flow of information or innovation (Granovetter 1973; Burt 2004). Individuals with either high degree and/or high centrality are likely to be keystone individuals with regard to controlling flow. In the context of disease, individuals with particularly high degree are termed "super-spreaders" (Meyers et al. 2005).
After using a SN analysis to help identify a keystone individual (Fewell 2003), further study might focus on the traits (e.g., behavioral type) of the keystone, and whether the individual is truly important, or whether the individual is merely the current occupant of a keystone role. In the latter case, if the individual was removed with no disruption of SNS, another individual could move quickly into that keystone role.

\section{Social networks carry over across contexts}

In many social systems, particularly those involving longterm associations, conventional wisdom suggests that a key outcome of social interactions is that they affect later interactions involving these, or in some cases, different individuals. That is, the SNS in one context carries over to influence the SNS in another context. For example, if we ask "why do males fight with each other early in the mating season?", the answer is often that the dominance relations established by male-male competition govern later access to mating females. That is, SNS in male-male competition influences the male-female mating SNS. Figure 3 shows a generalized view of SN carryovers across contexts influencing mating patterns. The simple idea is that the male-male SNS, the female-female SNS, and earlier M-F social interactions might together influence the male-female interaction pattern during the mating season that, in turn, has potentially important effects on the malefemale mating pattern. Our sense is that animal behaviorists have long thought about these social interactions in this way, but have generally not studied them using a sophisticated and formal quantitative framework. At the individual level, one can use SN metrics to characterize each male's SN traits (his degree, reach, centrality, etc.) during male-male interactions and then test whether these SN traits are correlated to male SN traits in male-female

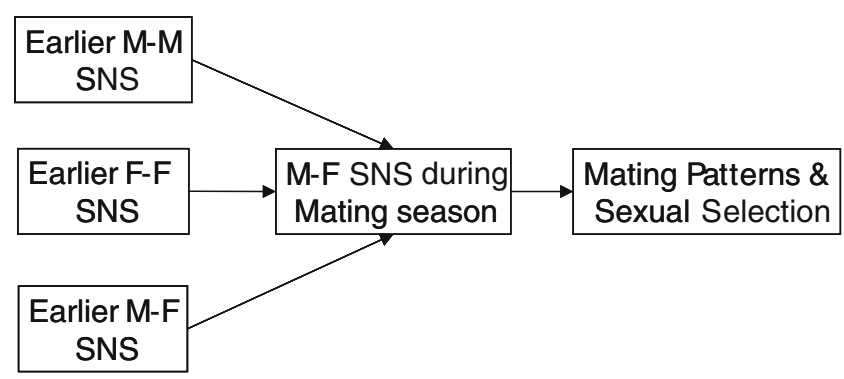

Fig. 3 Social network carryovers leading up to the pattern of mating success. The pattern of interactions (e.g., competitive or cooperative) among males (the M-M social network structure, SNS), among females (the F-F SNS), and between males and females (the M-F SNS) earlier in the season or during the mating season can carry over to govern male-female interactions during the mating season that determine patterns of mating success. These possibilities are well known but can be better quantified using SN metrics 
interactions and mating patterns. At a group level, one can examine whether variation in the group's overall SNS (mean male degree, clustering coefficient, or the degree of substructure) during the male-male competition phase is related to the subsequent pattern of male-female interaction and the resulting strength and direction of sexual selection on male behavior or morphology.

A conceptually parallel framework could be useful for studying carryovers in interactions between any two (or more) types of players: predators and prey, hawks and doves, producers and scroungers, or cooperators and defectors.

The notion of a multi-context framework featuring carryovers in SNS or SN traits across contexts is analogous to behavioral syndromes (aka animal personalities) which involve carryovers in behavioral type across contexts (Sih et al. 2004a, b). The importance of SN carryovers should depend on both the strength of the carryover per se, and the stability of the individual interactions. If SN carryovers are important, then traits that influence interactions either earlier or later in a different context could influence success in a particular focal context. For example, cooperative social networks established in an earlier phase could affect competitive success or mating preferences later on. Malefemale cooperation before the mating season could influence mate choices that affect later male-female cooperation in biparental care. Or, aggressiveness at an early time can determine dominance status that allows an individual to be an effective conflict mediator at a later time.

\section{Major issues in behavioral ecology}

The general point is that although social networks are clearly often structured and SNS often matters in ways that are part of our basic thinking about social biology, animal behaviorists often have not studied these issues quantitatively. SNT provides a sophisticated framework for quantifying SNS and thus for testing ideas on how SNS might influence social dynamics. Next, we discuss some specific insights that the SN approach offers for major issues in behavioral ecology.

Mate choice and sexual selection

The main points discussed above have major potential applications for mating behavior and sexual selection. First, as noted above, within a group, variation among females in number of males encountered (the female's cross-gender degree) should influence choosiness and thus sexual selection. Recent work on individual variation among females in mate preferences also shows that females vary in choosiness per se (Morris et al. 2003); some females exhibit strong mating preferences while others are relatively unselective. Choosiness could be a personality trait (Sih and Bell 2008; Sih et al. 2009), or might reflect variation in social experiences that reflect $\mathrm{SN}$ position. Females with high degree (i.e., that encounter many males) or centrality (e.g., that interact with males in multiple subgroups) might experience many high quality males, and are therefore more choosy. This idea also extends to differences among groups that might appear similar unless one quantifies the SNS. Comparing two groups of the same size, if individuals in one group are better connected (higher degree) than the other, females encounter more males to choose among, and should thus be choosier.

Indirect SN effects also play a key role in mating biology and sexual selection. As noted earlier, male fertilization success should depend on not just the number of female mating partners (male mating degree) but also the exclusivity of his mating. Low exclusivity should be associated with stronger sperm competition and/or a higher frequency of extrapair fertilizations. This point is obvious, yet few studies show this SN metric. Diagramming a mating network would identify not only each male's degree and exclusivity but also the specific pattern of which males are in competition for which females. As noted earlier, a key statistic should be the male-female degree correlation. If the two are positively correlated, then this dilutes the value of high mating success (as defined by number of matings) and reduces sexual selection. One mechanism that should tend to result in a positive correlation between male and female mating degree (and thus a negative correlation between male mating degree and exclusivity) is a tradeoff between time spent acquiring mates (increasing mating degree) versus guarding or providing incentives for mate fidelity (necessary to increase exclusivity). Another mechanism involves positive assortative mating between males and females that are more motivated to mate. These ideas are not new. The point is that SNT can play a useful role in providing quantitative assessment of these hypotheses.

In many systems, males exhibit alternative mating tactics, e.g., territorial vs. satellite males (Shuster and Wade 1991; Sinervo and Lively 1996; Watters 2005), with potentially complex SNS. For example, territorial males can differ from each other in their pattern of interaction with other territorial males, with satellite males and with females. If the same "hub" (high centrality) males that attract more females also must compete with more neighboring territorial males and more intruding satellite males (i.e., if there are positive degree correlations across these categories), then this can substantially reduce a hub male's mating success. Satellite males can also differ in their SN traits. Some might stay near one territorial male while others are sneakers that attempt to steal matings from many territorial males. SN metrics can quantify these 
differences in SN position and their relationships with mating success.

Finally, SN carryovers across contexts in a multi-context framework clearly play a major role in mating systems. One familiar idea is that earlier interactions in male-male competition set the stage for male-female social interactions that underlie male-female mating patterns. In the SN context, one might quantify the SNS and each individual's $\mathrm{SN}$ traits in each stage of this sequence to quantify carryovers across contexts. The usual notion is that male dominance in male-male competition governs his access to females and his later mating success. SNT potentially adds more detail to this picture by quantifying: (1) how dominance relates to various SN traits; (2) how dominance and other SN traits (e.g., degree, reach, centrality) relate to the pattern of access to females (the male-female interaction SNS); and (3) how the pattern of access to females relates to the mating SNS. While these theoretical frameworks fit our intuition on how these social interactions might work, SNT provides a useful tool that can bring quantitative behavioral ecology to bear on these issues in practice.

The first stage in this sequence need not be a male-male competition SN. It could be a male-male cooperative network or an earlier male-female interaction network. In some systems, it is plausible that earlier cooperative coalitions formed by males, or either cooperation or competition between individual males and females set the stage for later male-female mating interactions. Again, SNT provides a framework for studying these carryovers.

McDonald (2007) recently provided an exciting example of a surprising SN carryover in a mating context. He found that for long-tailed manakins where only a few, high status (alpha) males have opportunities to mate, the best predictor of attaining high mating status was not a male's current social network position but was instead his centrality in male-male interactions several years earlier. Apparently, young males that interact cooperatively across multiple leks, each with partially overlapping sets of 5-15 males, establish persistent connections that facilitate social rise to high status roughly 5 years later (also see McDonald 2009).

\section{Cooperation}

SNT also provides a quantitative framework for testing familiar ideas about cooperation. One mechanism that can favor cooperation is reciprocal altruism (Trivers 1971). Conventional wisdom suggests that a group's SNS should have important effects on the evolution of cooperation by reciprocal altruism. Theory suggests that as group size increases, cooperation should decrease (Suzuki and Akiyama 2005). Studies on humans (Hamburger et al. 1975; Louis et al. 2007) and rhesus monkeys (Berman et al.
1997) corroborate this prediction. For groups of a given size, cooperation is more likely to be favored if individuals interact in relatively small subgroups (e.g., primarily with a few neighbors; Santos et al. 2006). Recent theory formalized this intuition, showing that, given some simplifying assumptions, cooperation is favored if $b / c>k$, where $b$ and $c$ are the benefits and costs of the cooperative act, and $k$ is the average number of interacting neighbors in the social group, or in SN metrics, the group's mean degree (Ohtsuki et al. 2006). If all individuals in a group interact with each other, degree is large, and defectors tend to do better than cooperators. However, if the group is more finely structured (e.g., lower degree, higher clustering coefficients), this makes cooperation more likely. Examples of these networks are illustrated in Fig. 4.

Notably, the overall network's pattern of assortment by degree (measured by the degree correlation) can have important effects on cooperation. If individuals show positive assorting by degree (well-connected individuals interact with each other), this allows defectors to invade and reduces the likelihood of cooperation. In contrast, a SNS with negative degree correlations where numerous individuals interact primarily with a well-connected hub makes cooperation more likely (Rong et al. 2007). More studies showing data on the mean and distribution of degree in one or more social groups would be valuable.

In the social sciences, much of the $\mathrm{SN}$ discussion has revolved around "small-world" networks that could allow cooperation to persist even in larger social groups. "Small-world" networks feature dense clusters of interacting individuals within the larger network (Watts and Strogatz 1998) that could reduce the effective value of $k$ to a size that allows $b / c$ to exceed $k$ and thus promote cooperation. Links between distant individuals in a smallworld network might correspond to defectors who disrupt cooperation (see Fig. 4).

Social structure based on the traits of neighbors should also matter; e.g., individuals should be more cooperative with familiar neighbors. Croft et al. (2006) tested this idea using predator inspection in guppies (Poecilia reticulata). They confirmed that pairs of female fish that were often found together in the same, small schools tended to be more cooperative (switching leader vs. follower positions) while inspecting. Other familiar hypotheses that one can test using a SN framework include: (1) do cooperators tend to interact primarily with other cooperators? Is there a strategy $\times$ strategy correlation in the SN? (2) Within a group, is there a correlation between an individual's SN position and its tendency to give or receive favors? Are high degree individuals less (or more) cooperative? (3) Do individuals that sit between subgroups (individuals with high centrality) engage in hitrun cheating? 


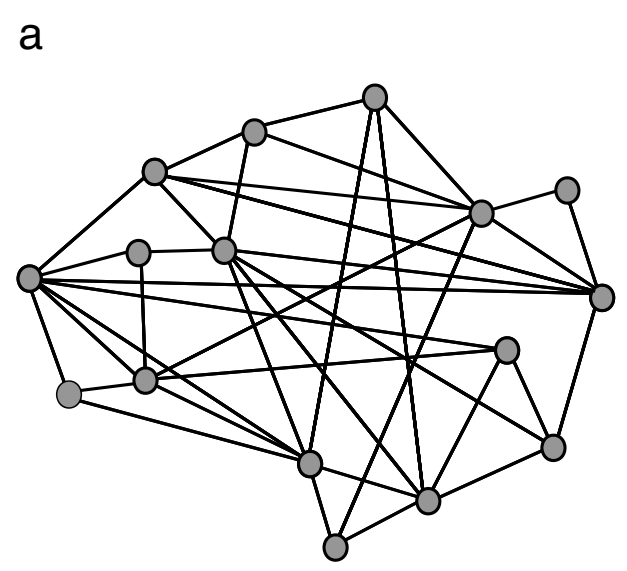

Fig. 4 Two examples of the same arrangements of nodes, but different arrangements of edges. If cooperation in this system has a cost, $c=1$ and a benefit, $b=4$, then $b / c=4$. Network $4 a$ would not promote cooperation because average degree $k=4.875$ which is $>b / c$, and most individuals interact with individuals across the entire network. Network $4 b$, an arrangement of edges that is more like that expected in a small world network, could support cooperation. The

Indirect effects (e.g., eavesdropping, policing) are often thought to be important in the evolution of reciprocal altruism. Simple questions about indirect SN links include: Does the SN position of a potential recipient influence the likelihood of a donor being altruistic? Are individuals more cooperative with recipients that are well connected? A possible benefit of this might arise if there are chains or loops of cooperation. A cooperative act towards X might not only increase the chance of reciprocation by $\mathrm{X}$ but also by other "friends" of X. Examples exist in the literature of individuals receiving benefits from higher ranking individuals by interacting with an associated middle individual, such as food sharing in stumptail macaques (Macaca arctoides) (Estrada et al. 1978). Conversely, being altruistic towards a well-connected individual might not be favored if the reciprocated benefits must be shared with others (i.e., if benefits are diluted).

For eavesdropping, SN metrics can potentially characterize not only whether someone is watching, but the average number of eavesdroppers, and most intriguingly, the SN position of eavesdroppers. Presumably information transferred to an eavesdropper is more important if the eavesdropper is well connected - if it has high degree, high reach, or high centrality. Are individuals more cooperative when they are being watched by well-connected eavesdroppers?

With regard to carryover effects, Croft et al. (2006) found that individuals that interacted frequently in the absence of predators also engaged more in predator inspection together; this represents a carryover from an apparently non-cooperative, safe context to a cooperative, dangerous context. Other potential SN carryovers include carryovers between SNS associated with different aspects

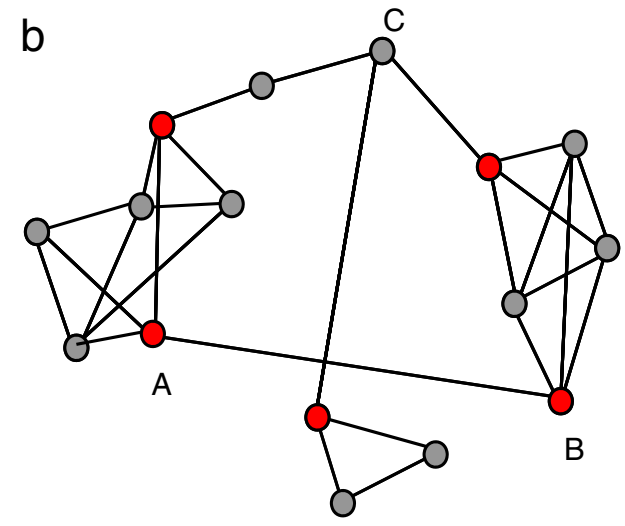

larger group is partitioned into smaller groups that interact closely. For this network $k=3.375$ which is $<b / c$. Red nodes indicate individuals that could be defectors from their subgroup and therefore disrupt cooperation. When considering information flow in this network, the red nodes could be individuals that would facilitate or control information flow in the network

of cooperation (grooming, conflict coalitions, cooperative foraging, resource sharing, or cooperative vigilance; de Waal 1997; Palagi et al. 2004; Gilby 2006; Schino 2007) and between cooperative social networks and mating networks (Palagi et al. 2006). For example, do females prefer to mate with males that cooperated with them earlier?

The other main mechanism, indeed probably the prevalent mechanism explaining cooperation in nature, is kin selection. Again, various hypotheses can be tested using SN metrics. Comparing groups, one could ask whether groups that differ in mean relatedness also differ in SNS - in mean degree, clustering, or pattern of substructure. If a group consists of largely unrelated individuals, do they cooperate in small, tight groups (low degree, high clustering; smallworld networks) as predicted by reciprocal altruism theory (Ohtsuki et al. 2006)? Conversely, if group members are primarily kin, do we see broader connectivity, particularly in the context of cooperation? Within groups, do communities (groups of individuals that are more connected to each other than to individuals in other groups) correspond to kinship groups (or not, see McDonald 2009)? At the individual level, are individuals that have higher mean relatedness to others in the group also well connected in general (high degree)?

A potentially fascinating indirect effect in kinship social networks involves in-law effects. Do individuals often connect and cooperate not only with kin, but with partners of kin?

Dominance hierarchies

Dominance hierarchies are a field where investigators have traditionally collected data on the pattern of interactions 
among individuals in a social group. Although some have expressed their results in the form of network-like diagrams, most studies focus on assigning dominance rankings and quantifying the linearity of the hierarchy. Dominance rankings are typically based on the pattern of winninglosing and not on the pattern of interaction. Interaction patterns, however, seem likely to be very important. For example, the influence of an alpha individual presumably depends on how much it interacts with others in the group. All else being equal, alpha individuals that are more interactive (high degree, high reach) should have larger direct and perhaps indirect effects. Whom the alpha interacts with should also be interesting. Does the alpha interact primarily with other high-ranking individuals, or does the alpha interact broadly with all members of the group? For other individuals, do they interact primarily with individuals above them in rank or primarily with lower-ranking individuals?

When analyzing aggressive interactions (or cooperative ones) associated with dominance, it is obviously important to distinguish between initiators and recipients; i.e., to identify directionality in the interaction. Although directional networks (that consider the polarity of the interaction proceeding from one individual to another) have been used in social science studies for years, they remain underrepresented in studies of animal networks (Newman 2003a). Unfortunately, the mathematics of directional networks is more complex and less well developed than undirected networks. Given that directionality is often important in animal interactions, further understanding of the statistical properties of directional networks is a key future direction for SNT.

Indirect effects (e.g., coalitions (Connor et al. 1992), coat-tail effects (Wiley 1990) such as parental effects on offspring rank) are often thought to be important in dominance relations. Are these indirect effects associated with individuals of a particular rank or SN position? Overall, do groups include reasonably distinct communities (cliques) and if so, how do interaction patterns differ between communities, and how might those differences be explained by key individuals in each community? These are mainstream issues in our thinking about dominance hierarchies that can be addressed in new, quantitative ways using SN metrics such as modularity, a measure of network division (Newman and Girvan 2004) and assortativity (Newman 2003b).

The tradition of focusing on rank rather than some more subtle aspect of the interaction patterns is based in part on an implicit carryover assumption - that ultimately, future access to resources, and more generally, costs and benefits of future competitive interactions, depend primarily on rank. This assumption can be tested quantitatively by comparing the relative or interactive effects of rank and other SN traits on future success. It would not be surprising, for example, to find that being well connected per se has benefits (or costs).

With regard to keystone individuals, an obvious possibility is that alpha individuals are particularly important. An intriguing alternative involves the existence of conflict mediators (Flack et al. 2005, 2006) who perform a policing function that reduces conflict and increases cooperation. Flack et al. (2005) showed that in pigtail macaques (Macaca nemestrina), the distribution of power within a social group influences the costs and benefits of policing that can, in turn, explain the existence of conflict mediators. Using a novel approach for SNT of comparing real experimental manipulations with "virtual experiments" (simulated social manipulations), Flack et al. (2006) performed a fascinating study where they temporarily removed a key conflict mediator and documented a shift in the overall group SNS towards reduced interaction (lower degree) and an increased tendency to interact in small clusters (high clustering coefficient, low reach) of similar individuals.

\section{Social learning and information flow}

In studies on humans, one major use of SNT involves analyses of how SNS influences flow, e.g., of information, innovation, or disease (Gould and Fernandez 1989; Meyers et al. 2005; Björneborn 2006; Rothenberg and Muth 2007). Studying analogous issues in non-human animals represents an obvious use of SNT in animal behavior. Krause et al. (2007) and Wey et al. (2007) recently reviewed ideas and extant studies applying SN metrics to study wildlife disease (Corner et al. 2003; Cross et al. 2004; Godfrey et al. 2009). For spread of information via social learning, relevant examples in animals (Galef and Laland 2005) might include song learning and tutoring (Smith et al. 2002; Beecher et al. 2007), mate copying (Godin et al. 2005; Valone 2007), learning of competitive styles (White et al. 2007), eavesdropping and reputational effects (Earley and Dugatkin 2002; Peake et al. 2002; Naguib et al. 2004; Valone 2007), public information about danger or resources (Coolen and Giraldeau 2003; Valone 2007), and spread of innovation (Reader and Laland 2000; Biro et al. 2003; Ottoni et al. 2005). SN issues include: (1) quantifying effects of the group's SNS on flow through the group. (2) Quantifying whether an individual's SN metrics predict its importance in flow. In particular, in the social sciences, a key issue involves the importance of individuals with high betweenness who bridge across "structural holes" between subgroups (e.g., Burt 2004), as illustrated in Fig. 4. (3) Examining correlations between individual traits and SN metrics, and how the interaction between these two might impact flow (e.g., Burt et al. 1998; Kalish and 
Robins 2006). Is flow particularly enhanced by individuals with a specific combination of personality traits and high betweenness?

An exciting area of study in social learning involves eavesdropping in communication networks (McGregor 2005; Valone 2007) - where individuals indirectly gain information by observing an interaction (communication) between other individuals. Eavesdroppers can gain information indirectly about the traits of other individuals (e.g., their resource holding potential, quality, behavioral type; Otter et al. 1999; Mennill et al. 2002; Naguib et al. 2004), about songs (e.g., song tutors; Beecher et al. 2007) or about the environment (e.g., about resources or risk). Experiments show that the information gained then influences later interactions; e.g., even with no direct interaction with potential opponents, males that have observed an earlier fight tend to approach and initiate attacks on previous losers sooner than previous winners (Oliveira et al. 1998; Earley and Dugatkin 2002; Dugatkin and Earley 2003), and eavesdropping females tend to prefer winning males (Otter et al. 1999; Doutrelant and McGregor 2000).

Public information obtained by eavesdropping could also establish new asymmetrical weak links in a network between individuals that have not yet interacted. These new weak links could later evolve into strong links when acted upon by an eavesdropper, as seen in male and female Siamese fighting fish, Beta splendens, that have observed males' aggression displays (Oliveira et al. 1998; Doutrelant and McGregor 2000), or male nightingales, Luscinia megarhynchos, that have heard vocal contests in neighboring territories (Naguib et al. 2004). As noted earlier, the SN approach can provide metrics to characterize the SNS of these communication networks. In turn, the SNS (e.g., pattern of who gains information from whom), and associated benefits and costs should influence the evolution of communication strategies (Dabelsteen 2004).

\section{Predator-prey interactions}

Many of the above points about cooperation, competition, or information flow should apply when predator-prey interactions involve social behaviors. Social foraging, for example, can involve cooperative hunting, information transfer about the location of high value food sources, or producer/scrounger interactions. Predator avoidance can involve group vigilance with information transfer (e.g., via alarm calls or predator inspection) or group defense. For each of these, SN analyses can quantify the pattern of interaction including both the SNS of the group, and each individual's SN metrics relative to these social interactions. As with other types of behavior, numerous hypotheses can be tested about the importance of group and individual SN metrics on individual foraging rates or survival. An interesting, little-explored connection between behavior and ecology should be how the group's SNS for predators and prey (and particularly the interaction between them) influences predation rates and overall predator-prey population dynamics. Prey availability could influence the predator's SNS (e.g., RamosFernandez et al. 2006; Henzi et al. 2009), predation risk could influence the prey's SNS, and both social network structures might influence predator-prey outcomes.

\section{Future directions}

Behavioral syndromes and social networks

An area of recent excitement in behavioral ecology involves the existence and importance of personalities or behavioral syndromes (Sih et al. 2004a, b). Behavioral syndromes exist when individuals show consistent differences in behavioral type (BT; e.g., boldness or aggressiveness) that can carry over across contexts. Sih et al. (2009) noted that an important next step should be further study of the social ecology of BTs. In the SN context, it should be interesting to quantify relationships between $\mathrm{BT}$ and $\mathrm{SN}$ position. An obvious possibility is that more sociable or more active individuals will have a higher degree. A perhaps less obvious possibility might be that bold individuals tend to bridge across otherwise separate social groups and thus have high centrality. Conversely, the impacts of key individuals in the SN (e.g., alpha individuals or high centrality individuals) should depend on their BT. In human business structures, a few studies have examined how the personality of high centrality individuals who bridge across separate groups influences the resulting flow of ideas and corporate efficiency (e.g., Kalish and Robins 2006; Burt et al. 1998). Studying parallel issues seems likely to be productive in studies of non-human animals.

As noted earlier, game theory assumes that individual fitnesses and group outcomes depend on the frequency of different behavioral types in the group (e.g., hawks-doves, producers-scroungers). Intuition suggests that social dynamics should depend on not just the mix of behavioral types in the group, but also on their pattern of interaction; e.g., on how SNS influences or reflects non-random patterns of interaction between individual hawks and doves. Adding SNS to game theory should prove valuable.

Space use and social networks

Spatial proximity might often play a large role in determining patterns of social interaction. All else the same, animals are presumably more likely to interact with individuals that are physically close by. In humans, for 
example, individuals are more likely to meet each other and thus interact if they share a workplace or school (Kossinets and Watts 2006). Within the SN framework, one might thus expect social proximity to mirror spatial proximity (e.g., McDonald 2009), particularly in relatively sedentary or territorial animals. Path length is a measure of social distance between any two individuals. Shorter path lengths correspond to greater social proximity. Alternatively, individuals might assort by various traits (e.g., age, size, rank, behavioral type, or genetic relatedness); social distance might then be correlated with one or more of these other traits (see McDonald 2009). The relative importance of these factors in determining SNS can thus be tested by comparing a group's matrix of SN distances with similarity matrices for other traits (e.g., using Mantel's tests).

If spatial proximity explains social proximity, then to understand SNS, we need to understand factors that influence patterns of space use (e.g., habitat preferences). In the mating context, in a classic paper, Emlen and Oring (1977) outlined ideas on how ecological (e.g., resource distributions and predation risk) and social factors might shape space use patterns that underlie interaction patterns that, in turn, influence sexual selection. SNT provides a framework for quantifying the SNS inherent in these dynamics.

Interestingly, recent theory suggests that non-random patterns of space use per se can produce apparently complex SNS. Ramos-Fernandez et al. (2006) modeled how the distribution of resources along with simple, sensible foraging movement rules (that have no explicit social component) can produce patterns of association that resemble fission-fusion societies. They characterized their model outputs in terms of both standard group metrics (e.g., group size) and SN metrics. The next step could be to add social interactions (e.g., partner choices that we discuss next) to examine how the combination of movement rules and social interaction rules determine SNS.

Partner choice and social network structure

Beyond quantifying SNS per se, a deeper understanding of SNS requires understanding mechanisms that determine the pattern of interactions. Who interacts with whom and why? In the previous section, we discussed how patterns of space use might influence SNS. Another obvious mechanism that can govern SNS is partner choice. Social scientists have made some progress on studying how active partner choice might influence the emergent SNS. Some extant models include simple negative versus positive feedbacks in partner choice; i.e., whether an interaction makes it more or less likely that individuals will interact again. A form of positive feedback is preferential attachment-the tendency for individuals to prefer to associate with individuals who already have many partners. This results in what SNT calls a scale-free degree distribution with a few individuals with very high degree and many with relatively low degree (i.e., with most individuals interacting primarily with hubs who have many partners; Barabási and Albert 1999; Newman 2003a). An example of this in behavioral ecology is mate copying. In contrast, negative feedback tends to result in a relatively uniform network that should be closer to the "mean field" assumption of simple models.

Other suggested partner choice mechanisms that can affect SNS include: (1) homophily (positive assortative partner choice; McPherson et al. 2001); (2) negative assortative partner choice (opposites attract); (3) triad closure - a tendency to become friends with friends of your friends (Kossinets and Watts 2006); and (4) a tendency for individuals to choose to span structural holes as a strategy to gain information or resources.

\section{Adaptive partner choice}

To date, models of SNS construction have rarely explicitly considered the benefits and costs of the partner choices that underlie SNS. In reality, individuals often exhibit adaptive partner choice. A critical area for future study is thus the development of game theory models of adaptive partner choice and SNS. Depending on the context, adaptive partner choices could depend on numerous partner traits (e.g., quality, health, resource holding potential, relatedness, aggressiveness, cooperativeness), and on the social (e.g., density, sex ratio) or ecological context (e.g., predation risk). A start could be models where one individual in each dyad controls whether a link forms or not. More complex models would allow both individuals to control the formation of a link-if either individual can break the link, both must "approve" for a link to form. An existing, relevant theoretical framework might be the transactional models of reproductive skew where a dyad forms when a dominant (who claims most of the benefits of the dyad through mating) gains from the presence of a subordinate but must offer incentives for the subordinate to stay (Reeve 2000). The most relevant models of this sort should be ones where both dominants and subordinates have alternative options - other partners that they can join. Modifying these models to explicitly include multiple individuals and the emergent SNS seems like a promising future direction.

Social scientists have done some analyses combining game theory and SNT (Skyrms and Permantle 2000) built, for example, around the prisoner's dilemma (Ebel and Bornoldt 2002; Zimmerman et al. 2004). A recent model showed that adaptive partner choice can result in a SNS with a scale-free degree distribution and positive degree correlations (Ren et al. 2006). A fascinating complexity is 
the possibility that benefits and costs depend on each potential partner's SN position (Holme and Ghoshal 2006); e.g., on the partner's network centrality. In that case, each link depends on other links formed by potential partners, and thus potentially on the entire SNS. Another interesting complexity involves the possibility that a communication network provides the information that shapes the SNS for some other sort of interaction (Rosvall and Sneppen 2005).

Finally, if the group's SNS (and other social group characteristics like density and sex ratio) influences fitness benefits of partner choice, then individuals should exhibit social group choice-not just social partner choice per se. And, if groups and interactions can be stable over long periods, adaptive partner and social group choice should be evaluated in a multi-context framework. Individuals might choose partners depending on their value in a current context (e.g., foraging cooperation) as well as future contexts (mating and parental care). Although understanding, modeling, and quantifying all this is obviously daunting, it is plausibly the reality of how social systems work.

\section{Dynamics and stability of social networks}

The above discussion implies that social networks should be dynamic. In contrast, applications of network theory (i.e., genetics, epidemiology, community ecology, social networks, etc.) usually assume that networks are static. An empiricist can take a snapshot of a network and then use SNT to project some outcome based on the assumption that the network will not change. In reality, network structure almost certainly changes, often due to adaptive "partner" choices. For example, epidemiological models with SNS typically examine how aspects of SNS (e.g., existence of super-spreaders or of distinct cliques) influence disease spread, assuming a static network. The fact that infected individuals might change their behavior (e.g., interact with fewer people), or that uninfected individuals might avoid infected ones is not usually included even though these possibilities seem obvious (but see Gross et al. 2006). Longitudinal studies are rare in the SNT literature even in humans (Kossinets and Watts 2006), yet these types of studies are essential to assessing the dynamic nature of networks. Questions to study within a dynamic framework of changing network linkages could include: (1) dynamic formation and development of SNS as individuals enter a group (Kossinets and Watts 2006); (2) equilibrium SNS and stability of SNS given particular mechanisms of partner choice; and (3) response of SNS to shifts in group membership, social group traits (e.g., density, sex ratio) or ecological factors (e.g., change in food abundance or distribution, change in predation risk).

An issue of great interest in some other applications of network theory involves the effects of network structure on network robustness or stability. How might the structure of a genetic or neural network make it more robust in the sense of being able to withstand changes (mutations, insertions, deletions) without change in function (Humphries et al. 2006)? How might the structure of the community (connectivity and more detailed aspects of connectivity) influence community stability (Dunne et al. 2004; Bascompte et al. 2006)? The analog in behavior has not received much study. How does SNS facilitate stability of the group's membership, roles, and interactions that make up the SNS? Does SNS introduce inertia that tends to constrain individual behavioral plasticity? Network constraint is a metric that roughly measures the strength of connection between a focal individual and all other individuals in the network via both direct and indirect links. Low network constraint means a less tight connection to an immediate group but potentially weak ties to different subgroups. A hypothesis is that an individual's network constraint value predicts its behavioral plasticity either in terms of behavioral type (aggressiveness, boldness) or its tendency to change SN position either spontaneously or in response to changing social or environmental conditions.

Some fascinating issues revolve around SN instability. Fission-fusion societies alternate between a few large groups and breakup into many, smaller groups (Symington 1990; Sundaresan et al. 2007). Lusseau and Newman (2004) showed, intriguingly, that in a bottlenose dolphin (Tursiops truncates) community in Scotland, one keystone individual that was the high centrality bridge between two subgroups apparently played a critical role in maintaining a connection between these two groups. When that individual left, the groups split apart, but fused again when the individual returned. This example highlights an important insight from SNT for stability of animal groups: not all individuals are equal in their influence on group cohesion. SNS may break down more rapidly with targeted removals of keystone individuals, an issue relevant to animal populations of conservation concern. Another example of group instability involves SNS breakdowns that result in chaos or warfare (e.g., in some rhesus macaque groups, McCowan et al. 2008). SN questions include-what conditions maintain group SNS and what facilitates group breakdown? Is group instability regulated by external factors (e.g., resources, risk) or by the SNS itself? If the latter, what SN group metrics predict the likelihood of alternative SNS? When do key individuals (e.g., conflict mediators or bridges) determine SNS and the transition between alternative SNS?

Social network structure and group selection

The notion that group SNS can influence average group performance or the stability of group performance suggests 
the possibility of group selection shaping SNS. Although group selection has not been popular in behavioral ecology, given the right conditions, it remains a potentially important force in the evolution of group and individual traits (Dugatkin and Reeve 1994; Wilson and Wilson 2007). This notion has long been a major part of the thinking in social insects where the pattern (potentially quantified using a SN approach) of connection and information transfer among groups doing different tasks (e.g., foraging, brood care) affects the emergence of collective behaviors that influence colony fitness (Fewell 2003). Keystone individuals (the queen, key foragers, dancers, vibrators) can, by definition, play a critical role in these group dynamics. As is often the case, we might expect a conflict between adaptive individual partner choices that increase individual fitnesses and the effects of these partner choices on group fitness. Using SN metrics to compare social dynamics and group performance across multiple groups should be rewarding.

\section{Final remarks}

Our focus has been on the exciting issues that can be addressed by the social network approach. We are aware that there are many, non-trivial methodological and statistical issues that must be tackled in order to strike the right balance between the need to capture enough of the complexity of multi-faceted interactions among individuals in a social group, and the need for a relatively simple, quantitative description of SNS. We refer the reader to other texts for discussion of these issues (e.g., Croft et al. 2008; James et al. 2009; Franks et al. 2009). The parallel exploration of new methods, new applications, and new theory should prove exciting and insightful.

Acknowledgements This paper emerged from a workshop on social network theory funded largely by the Animal Behavior Graduate Group at the University of California at Davis. It benefited greatly from interactions with members of the workshop group including: Jason Watters, Becky Fox, Paul Haverkamp, Evan Girvetz, Barbara Clucas, Maja Makagon, and Mark Lubell. Others who helped shape the paper include: Darren Croft, Jessica Flack, Daniel Promislow, and participants in the discussion at the IEC conference. The senior author also thanks Caitlin McGaw for invaluable, general insights on human networks and social interactions. This work was supported by National Science Foundation grants to A. Sih (IOS 0078033 and 0222063).

Open Access This article is distributed under the terms of the Creative Commons Attribution Noncommercial License which permits any noncommercial use, distribution, and reproduction in any medium, provided the original author(s) and source are credited.

\section{References}

Barabási AL, Oltvai ZN (2004) Network biology: understanding the cell's functional organization. Nat Rev Genet 5:101-113

Barabási AL, Albert R (1999) Emergence of scaling in random networks. Science 286:509-516

Bascompte J, Jordano P, Olesen JM (2006) Asymmetric coevolutionary networks facilitate biodiversity maintenance. Science 312:431-433

Beecher MD, Burt JM, O'Loghlen AL, Templeton CN, Campbell SE (2007) Bird song learning in an eavesdropping context. Anim Behav 73:929-935

Berman CM, Rasmussen KLR, Suomi SJ (1997) Group size, infant development and social networks in free-ranging rhesus monkeys. Anim Behav 53:405-421

Biro D, Inoue-Nakamura N, Yamakoshi G, Sousa C, Matsuzawa T (2003) Cultural innovation and transmission of tool use in wild chimpanzees: evidence from field experiments. Anim Cogn 6:213-223

Björneborn L (2006) 'Mini small worlds' of shortest link paths crossing domain boundaries in an academic Web space. Scientometrics 68:395-414

Burt RS, Jannotta JE, Mahoney JT (1998) Personality correlates of structural holes. Soc Netw 20:63-87

Burt RS (2004) Structural holes and good ideas. Am J Sociol 110:349-399

Coolen I, Giraldeau LA (2003) Incompatibility between antipredatory vigilance and scrounger tactic in nutmeg manikins, Lonchura punctulata. Anim Behav 66:657-664

Corner LAL, Pfeiffer DU, Morris RS (2003) Social-network analysis of Mycobacterium bovis transmission among captive brushtail possums (Trichosurus vulpecula). Prev Vet Med 59:147-167

Connor RC, Smolker RA, Richards AF (1992) Dolphin alliances and coalitions. In: Harcourt AH, de Waal FBM (eds) Coalitions and alliances in humans and other animals. Oxford University Press, Oxford, pp 415-443

Croft DP, James R, Thomas P, Hathaway C, Mawdsley D, Laland KN, Krause J (2006) Social structure and co-operative interactions in a wild population of guppies (Poecilia reticulata). Behav Ecol Sociobiol 59:644-650

Croft DP, James R, Krause J (2008) Exploring animal social networks. Princeton University Press, Princeton

Cross PC, Lloyd-Smith JO, Bowers JA, Hay CT, Hofmeyr M, Getz WM (2004) Integrating association data and disease dynamics in a social ungulate: bovine tuberculosis in African buffalo in the Kruger National Park. Ann Zool Fenn 41:879-892

Dabelsteen T (2004) Strategies that facilitate or counter eavesdropping on vocal interactions in songbirds. Anais da Academia Brasileira de Ciencias 76:274-278

de Waal FBM (1997) The chimpanzee's service economy: food for grooming. Evol Hum Behav 18:375-386

Doutrelant C, McGregor PK (2000) Eavesdropping and mate choice in female fighting fish. Behaviour 137:1655-1669

Dugatkin LA, Earley RL (2003) Group fusion: the impact of winner, loser, and bystander effects on hierarchy formation in large groups. Behav Ecol 14:367-373

Dugatkin LA, Reeve HK (1994) Behavioral ecology and levels of selection-dissolving the group selection controversy. Adv Study Behav 23:101-133

Dunne JA, Williams RJ, Martinez ND (2002) Food-web structure and network theory: the role of connectance and size. Proc Natl Acad Sci U S A 99:12917-12922

Dunne JA, Williams RJ, Martinez ND (2004) Network structure and robustness of marine food webs. Mar Ecol Prog Ser 273:291-302 
Ebel H, Bornoldt S (2002) Coevolutionary games on networks. Phys Rev E 66:056118

Earley RL, Dugatkin LA (2002) Eavesdropping on visual cues in green swordtail (Xiphophorus helleri) fights: a case for networking. Proc R Soc Lond B 269:943-952

Emlen ST, Oring LW (1977) Ecology, sexual selection, and the evolution of mating systems. Science 197:215-223

Estrada A, Sandoval JM, Manzolillo D (1978) Further data on predation by free ranging stump-tailed macaques Macaca arctoides. Primates 19:401-407

Fewell JH (2003) Social insect networks. Science 301:1867-1870

Flack JC, de Waal FBM, Krakauer D (2005) Social structure, robustness, and policing cost in a cognitively sophisticated species. Am Nat 165:E126-E139

Flack JC, Girvan M, de Waal FBM, Krakauer DC (2006) Policing stabilizes construction of social niches in primates. Nature 439:426-429

Franks DW, James R, Noble J, Ruxton GD (2009) A foundation for developing a methodology for social network sampling. Behav Ecol Sociobiol. doi:10.1007/s00265-009-0729-2

Galef BG, Laland KN (2005) Social learning in animals: empirical studies and theoretical models. Bioscience 55:489-499

Gilby IC (2006) Meat sharing among the Gombe chimpanzees: harassment and reciprocal exchange. Anim Behav 71:953-963

Godfrey S, Bull M, James R, Murray K (2009) Network structure and parasite transmission in a group living lizard, the gidgee skink, Egernia stokes. Behav Ecol Sociobiol. doi:10.1007/s00265-0090730-9

Godin JGJ, Herdman EJE, Dugatkin LA (2005) Social influences on female mate choice in the guppy, Poecilia reticulata: generalized and repeatable trait-copying behaviour. Anim Behav 69:9991005

Gould RV, Fernandez RM (1989) Structures of mediation: a formal approach to brokerage in transaction networks. Sociol Method 19:89-126

Granovetter MS (1973) The strength of weak ties. Am J Sociol 78:1360-1380

Gross T, Dommar D’Lima C, Blasius B (2006) Epidemic dynamics on an adaptive network. Phys Rev Lett 96-08701:1-4

Hahn MW, Kern AD (2005) Comparative genomics of centrality and essentiality in three eukaryotic protein-interaction networks. Mol Biol Evol 22:803-806

Hamburger H, Guyer M, Fox J (1975) Group size and cooperation. J Confl Resolut 19:503-531

Henzi, SP, Lusseau D, Weingrill T, van Schaik CP, Barrett L (2009) Cyclicity in the structure of female baboon social networks. Behav Ecol Sociobiol. doi:10.1007/s00265-009-0720-y

Hinde RA (1976) Interactions, relationships and social-structure. Man 11:1-17

Holme P, Ghoshal G (2006) Dynamics of networking agents competing for high centrality and low degree. Phys Rev Lett 96-098701:1-4

Humphries MD, Gurney K, Prescott TJ (2006) The brainstem reticular formation is a small-world, not scale-free, network. Proc R Soc Lond B 273:503-511

James R, Croft DP, Krause J (2009) Potential banana skins in animal social network analysis. Behav Ecol Sociobiol. doi:10.1007/ s00265-009-0742-5

Kalish Y, Robins G (2006) Psychological predispositions and network structure: the relationship between individual predispositions, structural holes, and network closure. Soc Netw 28:56-84

Kossinets G, Watts DJ (2006) Empirical analysis of an evolving social network. Science 311:88-90
Krause J, Croft DR, James R (2007) Social network theory in the behavioural sciences: potential applications. Behav Ecol Sociobiol 62:15-27

Krause J, Lusseau D, James R (2009) Animal social networks: an introduction. Behav Ecol Sociobiol. doi:10.1007/s00265-009-0747-0

Lee TL, Rinaldi NJ, Robert F, Odom DT, Bar-Joseph Z, Gerber GK, Hannett NM, Harbison CT, Thompson CM, Simon I, Zeitlinger J, Jennings EG, Murray HL, Gordon DB, Ren B, Wyrick JJ, Tagne J-B, Volkert TL, Fraenkel E, Gifford DK, Young RA (2002) Transcriptional regulatory networks in Saccharomyces cerevisiae. Science 298:799-804

Loughlin SB, Sejnowski TJ (2003) Communication in neuronal networks. Science 301:1870-1874

Louis KS, Holdsworth JM, Anderson MS, Campbell EG (2007) Becoming a scientist: the effects of work-group size and organizational climate. J High Educ 78:311-336

Lusseau D, Newman MEJ (2004) Identifying the role that animals play in their social networks. Proc R Soc Lond B (Suppl) 271:S477-S481

Lusseau D, Wilson B, Hammond PS, Grellier K, Durban JW, Parsons KM, Barton TR, Thompson PM (2006) Quantifying the influence of sociality on population structure in bottlenose dolphins. J Anim Ecol 75:14-24

McCowan B, Anderson K, Heagarty A, Cameron A (2008) Utility of social network analysis for primate behavioral management and well-being. Appl Anim Behav Sci 109:396-405

McDonald DB (2007) Predicting fate from early connectivity in a social network. Proc Natl Acad Sci U S A 104:10910-10914

McDonald DB (2009) Young-boy networks without kin clusters in a lek-mating manakin. Behav Ecol Sociobiol. doi:10.1007/s00265009-0722-9

McElreath R, Boyd R (2007) Mathematical model of social evolution. A guide for the perplexed. University of Chicago Press, Chicago

McGregor PK (2005) Animal communication networks. Cambridge University Press, New York

McPherson M, Smith-Lovin L, Cook JM (2001) Birds of a feather: homophily in social networks. Annu Rev Sociology 27:415-444

Mennill DJ, Ratcliffe LM, Boag PT (2002) Female eavesdropping on male song contests in songbirds. Science 296:873

Meyers LA, Pourbohloul B, Newman MEJ, Skowroski DM, Brunham RC (2005) Network theory and SARS: predicting outbreak diversity. J Theor Biol 232:71-81

Morris MR, Nicoletto PF, Hesselman E (2003) A polymorphism in female preference for a polymorphic male trait in the swordtail fish Xiphophorus cortezi. Anim Behav 65:45-52

Naguib M, Amrhein V, Kunc HP (2004) Effects of territorial intrusions on eavesdropping neighbors: communication networks in nightingales. Behav Ecol 15:1011-1015

Newman MEJ (2003a) The structure and function of complex networks. SIAM Rev 45:167-256

Newman MEJ (2003b) Mixing patterns in networks. Phys Rev E 67:026126.1-026126.13

Newman MEJ, Girvan M (2004) Finding and evaluating community structure in networks. Phys Rev E 68:26113.1-26113.15

Ohtsuki H, Hauert C, Lieberman E, Nowak MA (2006) A simple rule for the evolution of cooperation on graphs and social networks. Nature 441:502-505

Olesen JM, Bascompte J, Dupont YL, Jordano P (2006) The smallest of all worlds: pollination networks. J Theor Biol 240:270-276

Oliveira RF, McGregor PK, Latruffe C (1998) Know thine enemy: fighting fish gather information from observing conspecific interactions. Proc R Soc Lond B 265:1045-1049

Otter K, McGregor PK, Terry AMR, Burford FRL, Peake TM, Dabelsteen T (1999) Do female great tits (Parus major) assess 
males by eavesdropping? A field study using interactive song playback. Proc R Soc Lond B 266:1305-1309

Ottoni EB, de Resende BD, Izar P (2005) Watching the best nutcrackers: what capuchin monkeys (Cebus paella) know about others' tool-using skills. Anim Cogn 8:215-219

Palagi E, Cordoni G, Tarli SMB (2004) Immediate and delayed benefits of play behaviour: new evidence from chimpanzees (Pan troglodytes). Ethology 110:949-962

Palagi E, Paoli R, Tarli SB (2006) Short-term benefits of play behavior and conflict prevention in Pan paniscus. Int J Primatol 27:1257-1270

Peake TM, Terry AMR, McGregor PK, Dabelsteen T (2002) Do great tits assess rivals by combining direct experience with information gathered by eavesdropping? Proc R Soc Lond B 269:1925-1929

Polis GA, Strong DR (1996) Food web complexity and community dynamics. Am Nat 147:813-846

Proulx SR, Promislow DEL, Phillips PC (2005) Network thinking in ecology and evolution. Trends Ecol Evol 20:345-353

Ramos-Fernandez G, Boyer D, Gomez VP (2006) A complex social structure with fission-fusion properties can emerge from a simple foraging model. Behav Ecol Sociobiol 60:536-549

Reader SM, Laland KN (2000) Diffusion of foraging innovations in the guppy. Anim Behav 60:175-180

Reeve HK (2000) A transactional theory of within-group conflict. Am Nat 155:365-382

Ren, J, Wu X, Chang W, Chen G, Wang B (2006) Interplay between evolutionary game and network structure: the coevolution of social net, cooperation and wealth. Archiv. Phys. ArXiv:physics/0605250v2

Rong Z, Li X, Wang X (2007) Roles of mixing patterns in cooperation on a scale-free networked game. Phys Rev E 76:027101. doi:10.1103/PhysRevE.76.027101

Rosvall M, Sneppen K (2005) Self-assembly of information in networks. Europhys Lett 74:1109-1115

Rothenberg R, Muth SQ (2007) Large-network concepts and smallnetwork characteristics: fixed and variable factors. Sex Transm Dis 34:604-612

Santos FC, Pacheco JM, Lenaerts T (2006) Cooperation prevails when individuals adjust their social ties. PLOS Comput Biol 2:e140. doi:10.1371/journal.pcbi.0020140

Schino G (2007) Grooming and agonistic support: a meta-analysis of primate reciprocal altruism. Behav Ecol 18:115-120

Scott J (2000) Social network analysis. Sage, London

Shuster SM, Wade MJ (1991) Equal mating success among male reproductive strategies in a marine isopod crustacean. Nature 350:606-610

Sih A, Bell AM, Johnson JC, Ziemba RE (2004a) Behavioral syndromes: an integrative overview. Q Rev Biol 79:241-277

Sih A, Bell AM, Johnson JC (2004b) Behavioral syndromes: an ecological and evolutionary overview. Trends Ecol Evol 19:372-378

Sih A, Bell AM (2008) Insights for behavioral ecology from behavioral syndromes. Adv Study Behav 38:227-281
Sih A, Bell AM, Johnson JC (2009) Behavioral syndromes. In: Westneat DF, Fox C (eds) Evolutionary Behavioral Ecology, in press

Sih A, Watters JV (2005) The mix matters: behavioural types and group dynamics in water striders. Behaviour 142:1417-1431

Sinervo B, Lively CM (1996) The rock-paper-scissors game and the evolution of alternative male strategies. Nature 380:40-43

Skyrms B, Permantle R (2000) A dynamic model of social network formation. Proc Natl Acad Sci U S A 97:9340-9346

Smith VA, King AP, West MJ (2002) The context of social learning: association patterns in a captive flock of brown-headed cowbirds. Anim Behav 63:23-35

Sporns O, Kotter R (2004) Motifs in brain networks. PLOS Biol 2: e369. doi:10.1371/journal.pbio.0020369

Stumpf MPH, Kelly WP, Thorne T, Wiuf C (2007) Evolution at the system level: the natural history of protein interaction networks. Trends Ecol Evol 22:366-373

Suzuki S, Akiyama E (2005) Reputation and the evolution of cooperation in sizable groups. Proc R Soc Lond B 272:1373-1377

Sundaresan SR, Fischoff IR, Dushoff J, Rubenstein DI (2007) Network metrics reveal differences in social organization between two fission-fusion species, Grevy's zebra and onager. Oecologia 151:140-149

Symington MM (1990) Fission-fusion social organization in Ateles and Pan. Int J Primatol 11:47-61

Trivers RL (1971) The evolution of reciprocal altruism. Quarterly Review of Biology 46:35-57

Valone TJ (2007) From eavesdropping on performance to copying the behavior of others: a review of public information use. Behav Ecol Sociobiol 62:1-14

Watters JW (2005) Can the alternative male tactics 'fighter' and 'sneaker' be considered 'coercer' and 'cooperator' in coho salmon? Anim Behav 70:1055-1062

Watts DJ, Strogatz S (1998) Collective dynamics of 'small-world' networks. Nature 393:440-442

Wey T, Blumstein DT, Shen W, Jordan W (2007) Social network analysis of animal behaviour: a promising tool for the study of sociality. Anim Behav 75:333-344

White DJ, Gros-Louis J, King AP, Papakhian MA, West MJ (2007) Constructing culture in cowbirds (Molothrus ater). J Comp Psych 121:113-122

Whitehead H (1997) Analysing animal social structure. Anim Behav 53:1053-1067

Wiley RH (1990) Prior-residence and coat-tail effects in dominance relationships of male dark-eyed juncos, Junco hyemalis. Anim Behav 40:587-596

Wilson DS, Wilson EO (2007) Rethinking the theoretical foundation of sociobiology. Q Rev Biol 82:327-348

Yodzis P (2000) Diffuse effects in food webs. Ecology 81:261-266

Zimmerman MG, Eguiluz VM, San Miguel M (2004) Coevolution of dynamical states and interactions in dynamic networks. Phys Rev E 96-065102:1-4 